\title{
THE WEYL QUANTIZATION AND THE QUANTUM GROUP QUANTIZATION OF THE MODULI SPACE OF FLAT $S U(2)$-CONNECTIONS ON THE TORUS ARE THE SAME
}

\author{
RĂZVAN GELCA AND ALEJANDRO URIBE
}

\begin{abstract}
We prove that, for the moduli space of flat $S U(2)$-connections on the 2-dimensional torus, the Weyl quantization and the quantization using the quantum group of $S L(2, \mathbb{C})$ are the same. This is done by comparing the matrices of the operators associated by the two quantizations to cosine functions. We also discuss the $*$-product of the Weyl quantization and show that it satisfies the product-to-sum formula for noncommutative cosines on the noncommutative torus.
\end{abstract}

\section{Contents}

1. Introduction 2

2. The phase space we are quantizing 3

3. Review of the quantum group approach 4

3.1. Path integrals 4

3.2. Quantization using the quantum group of $S L(2, \mathbb{C})$

4. The Weyl quantization 8

$\begin{array}{lll}4.1 . & \text { The line bundle } & 8\end{array}$

4.2. The Hilbert space of the quantization 9

$\begin{array}{lll}4.3 . & \text { The operators of the quantization } & 11\end{array}$

5. Weyl quantization versus quantum group quantization 11

$\begin{array}{ll}\text { 6. The star product } & 14\end{array}$

\begin{tabular}{lll}
\hline 6.1. & Definition of the star product & 14
\end{tabular}

\begin{tabular}{lll}
\hline 6.2. & The generalized Hardy space & 15
\end{tabular}

\begin{tabular}{ll}
\hline 7. Final remarks & 17
\end{tabular}

\begin{tabular}{ll}
\hline References & 17
\end{tabular}

Date: January 16, 2001.

1991 Mathematics Subject Classification. 81S10, 81R50, 57R56, 81T45, 57M25.

Key words and phrases. Weyl quantization, quantum groups, topological quantum field theory, moduli spaces of flat connections, Jones polynomial, *-product. 


\section{INTRODUCTION}

Quantization is a procedure for replacing functions on the phase space of a physical system (classical observables) by linear operators. While understood in many general situations, this procedure is far from being algorithmic. Some more exotic spaces whose quantizations are of interest to mathematicians are the moduli spaces of flat connections on a surface. Among them the case of the moduli space of flat $S U(2)$-connections on a torus is a particularly simple example of an algebraic variety that fails to be a manifold, yet is very close to being one.

In this paper we compare two methods of quantizing the moduli space of flat $S U(2)$-connections on the torus. The first is the quantization using the quantum group of $S L(2, \mathbb{C})$. This quantization scheme arose when Reshetikhin and Turaev constructed a topological quantum field theory that explains the Jones polynomial of a knot. It describes both the quantum observables and the Hilbert spaces in terms of knots and links colored by representations of the quantum group of $S L(2, \mathbb{C})$. Heuristically, the operators of the quantization were defined by Witten using path integrals for the Chern-Simons action.

On the other hand, the moduli space of flat $S U(2)$-connections on the torus is the same as the character variety of $S U(2)$-representations of its fundamental group, so it admits a covering by the plane. Therefore we can apply a classical quantization procedure of the plane in an equivariant manner to obtain a quantization of the moduli space. The first such procedure was introduced by Hermann Weyl. It assigns to each smooth function (classical observable) on the plane the pseudo-differential operator with symbol equal to the function.

Our main result is the following

Theorem. The Weyl quantization and the quantum group quantization of the moduli space of flat $S U(2)$-connections on the torus are unitarily equivalent.

The paper is structured as follows. In Section 2 we describe the geometric realization and the Kähler structure of the moduli space of flat $S U(2)$ connections on the torus. In Section 3 we review Witten's description of the quantization for the particular case of the torus with a path integral of the Chern-Simons action, then explain the rigorous construction of Reshetikhin and Turaev using quantum groups. Here we also mention a result of the first author that describes the matrices of the operators associated to cosine functions in a distinguished basis of the Hilbert space. This distinguished basis consists of the colorings of the core of the solid torus by irreducible representations.

We then explain in detail the Weyl quantization of the moduli space (Section (4). This is done in the holomorphic setting, which can be related to the classical, real setting through the Bargman transform. A distinguished basis of the Hilbert space is introduced in terms of odd theta functions. Section 5 contains the main result of the paper (Theorem 5.3). It shows 
that the two quantizations are unitarily equivalent. The unitary equivalence maps the basis consisting of odd theta functions to the basis consisting of the colored cores of the solid torus. In Section 6 we discuss the $*$-product that arises from this quantization. We conclude with some final remarks about the quantization scheme based on the Kauffman bracket skein module, for which the result does not hold due to a sign obstruction.

\section{The phase Space We are quantizing}

Throughout the paper $\mathbb{T}^{2}$ will denote the 2-dimensional torus. The moduli space of flat $S U(2)$-connections on a surface is the same as the character variety of $S U(2)$-representations of the fundamental group of the surface [2], i.e. the set of the morphisms of the fundamental group of the surface into $S U(2)$ modulo conjugation. This is a complex algebraic variety. In the case of the torus, morphisms from $\pi_{1}\left(\mathbb{T}^{2}\right)=\mathbb{Z} \oplus \mathbb{Z}$ to $S U(2)$ are parameterized by the images of the two generators of $\mathbb{Z} \oplus \mathbb{Z}$, i.e. by two commuting matrices. The two matrices can be simultaneously diagonalized. Moreover conjugation can permute simultaneously the entries in the two diagonal matrices. Therefore the character variety is

$$
X(\mathbb{Z} \oplus \mathbb{Z})=\{(s, t) \quad|\quad| s|=| t \mid=1\} /(s, t) \sim(\bar{s}, \bar{t}) .
$$

This set is called the "pillow case". It has a $2-1$ covering by the torus, with branching points $(1,1),(1,-1),(-1,1),(-1,-1)$. We will think of the character variety as the quotient of the complex plane by the group $\Lambda$ generated by the translations $z \rightarrow z+1$ and $z \rightarrow z+i$, and by the symmetry with respect to the origin, which we denote by $\sigma$.

Off the four singularities $X(\mathbb{Z} \oplus \mathbb{Z})$ is a Kähler manifold, with Kähler form $\omega$ induced by $i \pi d z \wedge d \bar{z}$ on $\mathbb{C}$. Note that

$$
i \pi d z \wedge d \bar{z}=-2 i \partial \bar{\partial} \ln h(z, \bar{z}),
$$

where $h(z, \bar{z})=e^{-\frac{\pi}{2}|z|^{2}}$ is the weight of the Bargman measure on the plane. Also, note that the Kähler form $\omega$ is the genus one case of Goldman's symplectic form defined in 10 .

The classical observables are the $C^{\infty}$ functions on this variety. Using the covering map we identify the algebra of observables on the character variety with the algebra of functions on $\mathbb{C}=\mathbb{R} \oplus \mathbb{R}$ generated by $\cos 2 \pi(p x+q y)$, $p, q \in \mathbb{Z}$.

Another family of important functions on the character variety are $\sin 2 \pi n(p x+$ $q y) / \sin 2 \pi(p x+q y)$ where $p$ and $q$ are coprime. As functions on the moduli space, these associate to a connection the trace in the $n$-dimensional irreducible representation of $S U(2)$ of the holonomy of the connection around the curve of slope $p / q$ on the torus.

To quantize $X(\mathbb{Z} \oplus \mathbb{Z})$ means to replace classical observables $f$ by linear operators $o p(f)$ on some Hilbert space, satisfying Dirac's conditions:

1. $o p(1)=I d$,

2. $o p(\{f, g\})=\frac{1}{i \hbar}[o p(f), o p(g)]+O(\hbar)$. 
Here $\{f, g\}$ is the Poisson bracket induced on $X(\mathbb{Z} \oplus \mathbb{Z})$ by the form $\omega$, which is nothing but

$$
\{f, g\}=\frac{1}{i \pi}\left(\frac{\partial f}{\partial x} \frac{\partial g}{\partial y}-\frac{\partial f}{\partial y} \frac{\partial g}{\partial x}\right) .
$$

Also $[A, B]$ is the commutator of operators, and $\hbar$ is Planck's constant.

Since in our case the phase space is an orbifold covered by the plane, and this orbifold is Kähler off singularities, it is natural to perform equivariant quantization of the plane. We do this using Weyl's method and then compare the result with the quantization from [16] which was done using the quantum group of $S L(2, \mathbb{C})$.

\section{REVIEW OF THE QUANTUM GROUP APPROACH}

The quantization of the moduli space of flat $S U(2)$-connections on a surface performed using the quantum group of $S L(2, \mathbb{C})$ at roots of unity is an offspring of Reshetikhin and Turaev's construction of quantum invariants of 3-manifolds [16]. Their work was inspired by Witten's heuristic explanation of the Jones polynomial using Chern-Simons topological quantum field theory. We present below Witten's idea for the particular case of the torus, and then show how the Reshetikhin-Turaev construction yields a quantization of $X(\mathbb{Z} \oplus \mathbb{Z})$.

3.1. Path integrals. In [20], Witten outlined a way of quantizing the moduli space of flat connections on a trivial principal bundle with gauge group a simply connected compact Lie group. Let us recall how this is done when the group is $S U(2)$ and the principal bundle lies over the cylinder over the torus $M=\mathbb{T}^{2} \times[0,1]$.

For $A$ an $S U(2)$-connection on $M$ define the Chern-Simons Lagrangian to be

$$
\mathcal{L}=\frac{1}{4 \pi} \int_{M} \operatorname{tr}\left(A \wedge d A+\frac{2}{3} A \wedge A \wedge A\right)
$$

where tr is the trace on the 2-dimensional irreducible representation of $s u(2)$. The Lagrangian is invariant under gauge transformations up to the addition of an integer.

The fields we quantize are the flat connections on $\mathbb{T}^{2}$, and symplectic reduction restricts our attention to the moduli space of flat connections [2], hence to the character variety. Now, using Witten's idea we will associate operators to the observables of the form $2 \cos 2 \pi(p x+q y)$ and $\sin 2 \pi n(p x+$ $q y) / \sin 2 \pi(p x+q y)$ on the torus $p, q, n \in \mathbb{Z}$.

Let $N$ be some fixed integer called the level of the quantization. As such, Planck's constant is $\hbar=\frac{1}{N}$.

Assume that $p$ and $q$ are arbitrary integers, and let $n$ be their greatest common divisor. Denote $p^{\prime}=p / n, q^{\prime}=q / n$. Consider the cylinder over the torus $\mathbb{T}^{2} \times[0,1]$ and let $C$ be the curve of slope $p^{\prime} / q^{\prime}$ in $\mathbb{T}^{2} \times\left\{\frac{1}{2}\right\}$. Then the operator associated by the quantization to the function 
$\sin 2 \pi n\left(p^{\prime} x+q^{\prime} y\right) / \sin 2 \pi\left(p^{\prime} x+q^{\prime} y\right)$ and denoted shortly by $S(p, q)$ ( $S$ from sine) is determined by the following path integral

$$
<S(p, q) A_{1}, A_{2}>=\int_{\mathcal{M}_{A_{1}, A_{2}}} e^{i N \mathcal{L}(A)} \operatorname{tr}_{V^{n}}\left(\operatorname{hol}_{C}(A)\right) \mathcal{D} A
$$

where $A_{1}, A_{2}$ are conjugacy classes of flat connections on $\mathbb{T}^{2}, A$ is a connection on $\mathbb{T}^{2} \times[0,1]$ such that $\left.A\right|_{\mathbb{T}^{2} \times\{0\}}=A_{1}$ and $\left.A\right|_{\mathbb{T}^{2} \times\{1\}}=A_{2}$, and $\operatorname{tr}_{V^{n}}\left(\operatorname{hol}_{C}(A)\right)$, known as the Wilson line, is the trace of the $n$-dimensional irreducible representation of $S U(2)$ evaluated on the holonomy of $A$ around $C$ of slope $p^{\prime} / q^{\prime}$. Here the "average" is taken over all conjugacy classes of connections modulo the gauge group.

With the same notations one defines the operator $C(p, q)(C$ from cosine) representing the quantization of the function $2 \cos 2 \pi(p x+q y)$ by

$$
<C(p, q) A_{1}, A_{2}>=\int_{\mathcal{M}_{A_{1}, A_{2}}} e^{i N \mathcal{L}(A)}\left(\operatorname{tr}_{V^{n+1}}-\operatorname{tr}_{V^{n-1}}\right)\left(\operatorname{hol}_{C}(A)\right) \mathcal{D} A .
$$

We only discuss briefly the Hilbert space of the quantization assuming the reader is familiar with [1] and [20]. Next section will make these ideas precise. The Hilbert space is spanned by the quantum invariants (i.e. partition functions) of all 3-manifolds with boundary equal to the torus. Since any 3-manifold can be obtained by performing surgery on a link that lies in the solid torus, it follows that the Hilbert space of the quantization is spanned by the partition functions of pairs of the form $\left(S^{1} \times \mathbb{D}^{2}, L\right)$, where $L$ is a (colored) link in the solid torus $S^{1} \times \mathbb{D}^{2}$.

3.2. Quantization using the quantum group of $S L(2, \mathbb{C})$. The quantization of the character variety of the torus using the quantum group of $S L(2, \mathbb{C})$ is a particular consequence of the topological quantum field theory constructed in [16]. Let us briefly describe it.

Fix a level $r \geq 3$ of the quantization, and let $t=e^{\frac{\pi i}{2 r}}$. Comparing with previous section, $N=2 r$. Quantized integers are defined by the formula $[n]=\left(t^{2 n}-t^{-2 n}\right) /\left(t^{2}-t^{-2}\right)$. The quantum algebra of $s l(2, \mathbb{C})$, denoted by $\mathbb{U}_{t}$ is a deformation of its universal enveloping algebra and has generators $X, Y, K$ subject to the relations

$$
\begin{aligned}
& K X=t^{2} X K, \quad K Y=t^{-2} Y K, \quad X Y-Y X=\frac{K^{2}-K^{-2}}{t^{2}-t^{-2}}, \\
& X^{r}=Y^{r}=0, \quad K^{4 r}=1 .
\end{aligned}
$$

This algebra is Hopf, so its representations form a ring under the operations of direct sum and tensor product. Reducing modulo summands of quantum trace zero, this ring contains a subring generated by finitely many irreducible representations $V^{1}, V^{2}, \ldots, V^{r-1}$. Here $V^{k}$ has dimension $k$, basis 
$e_{-(k-1) / 2}, e_{-(k-3) / 2} \ldots, e_{(k-1) / 2}$ and the action of $\mathbb{U}_{t}$ is defined by

$$
\begin{aligned}
X e_{j} & =[m+j+1] e_{j+1} \\
Y e_{j} & =[m-j+1] e_{j-1} \\
K e_{j} & =t^{2 j} e_{j} .
\end{aligned}
$$

The idea originating in [14] and further developed in [16] is to color any link in a 3 -dimensional manifold by such irreducible representations. For a knot $K$ we denote by $V^{n}(K)$ its coloring by $V^{n}$. There is a rule, for which we refer the reader to [14] and [16], for associating numerical invariants to colored links in the 3 -sphere. Briefly, the idea is to use a link diagram such as the one in Fig. 1, with the local maxima, minima and crossings separated by horizontal lines, and then define an automorphism of $\mathbb{C}$ by associating to minima maps of the form $\mathbb{C} \rightarrow V^{n} \otimes V^{n}$, to maxima maps of the form $V^{n} \otimes V^{n} \rightarrow \mathbb{C}$, to crossings the quasitriangular $R$ matrix of the quantum algebra in the $n$-dimensional irreducible representation, and to parallel strands tensor products of representations. The automorphism is then the multiplication by a constant and the link invariant is equal to that constant. For $K$ a knot in the 3 -sphere, $V^{2}(K)$ is its Jones polynomial [13] evaluated at the specific root of unity, and for $n \geq 2, V^{n}(K)$ is called the colored (or generalized) Jones polynomial.

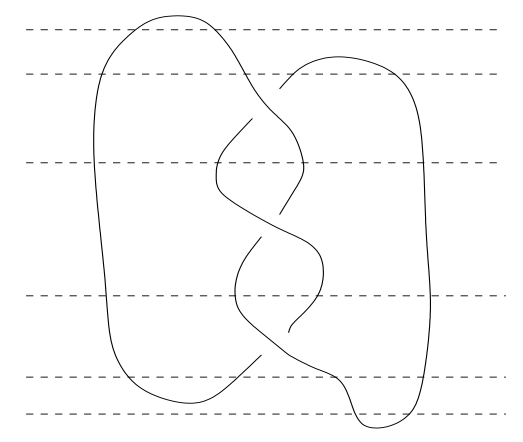

Figure 1.

We are now able to describe the quantization of the torus. First consider the vector space freely spanned by all colored links in the solid torus. On this vector space consider the pairing $[\cdot, \cdot]$ induced by the operation of gluing two solid tori such that the meridian of the first is identified with the longitude of the second and vice versa, as to obtain a 3-sphere (Fig. 2). The pairing of two links $\left[L_{1}, L_{2}\right]$ is equal to the quantum invariant of the resulting link in the 3 -sphere. The Hilbert space of the quantization is obtained by factoring the vector space by all linear combinations of colored links $\lambda$ such that $\left[\lambda, \lambda^{\prime}\right]=0$ for any $\lambda^{\prime}$ in the vector space. This quotient, denoted by $V\left(\mathbb{T}^{2}\right)$ by quantum topologists, is finite dimensional.

Let $\alpha$ be the core $S^{1} \times\{0\}$ of the solid torus $S^{1} \times \mathbb{D}, \mathbb{D}=\{z,|z| \leq 1\}$. A basis of $V\left(\mathbb{T}^{2}\right)$ is given by $V^{k}(\alpha), k=1,2, \ldots, r-1$. The inner product is 

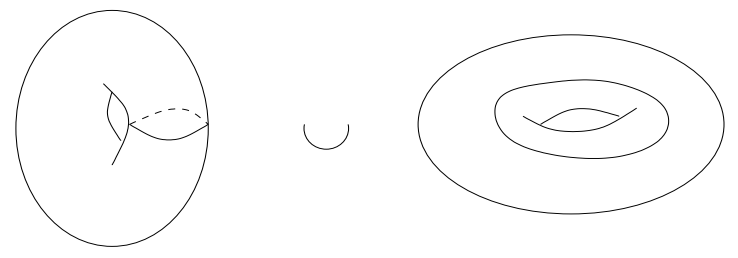

Figure 2.

determined by requiring that this basis is orthonormal. The pairing $[\cdot, \cdot]$ is not the inner product.

Let us point out that these basis elements play an important role in the construction of the Reshetikhin-Turaev invariants of closed 3-manifolds. Indeed, as suggested in [20] and rigorously done in [16], the quantum invariant of a 3-manifold obtained by performing surgery on a link is calculated by gluing to the complement of the link solid tori with cori colored by irreducible representations, computing the colored link invariants and then summing over all possible colorings.

Consider now two integers $p$ and $q$, let $n$ be their greatest common divisor, and let also $p^{\prime}=p / n, q^{\prime}=q / n$. The operator $S(p, q)$ associated to the classical observable $\sin 2 \pi n\left(p^{\prime} x+q^{\prime} y\right) / \sin 2 \pi\left(p^{\prime} x+q^{\prime} y\right)$ is obtained by coloring the curve of slope $p^{\prime} / q^{\prime}$ in the cylinder over the torus by the representation $V^{n}$. Also, as explained in the previous section, the operator that quantizes $2 \cos 2 \pi(p x+q y)$ is

$C(p, q)=C\left(n p^{\prime}, n q^{\prime}\right)=S\left((n+1) p^{\prime},(n+1) n q^{\prime}\right)-S\left((n-1) p^{\prime},(n-1) q^{\prime}\right)$.

Their action on the Hilbert space of the solid torus is defined by gluing the cylinder over the torus to the solid torus. The operator associated to an arbitrary function in $C^{\infty}(X(\mathbb{Z} \oplus \mathbb{Z}))$ is defined by approximating the function with trigonometric polynomials in cosines, quantizing those, then passing to the limit.

The matrices of the action can be computed using the pairing $[\cdot, \cdot]$, which now is nondegenerate on $V\left(\mathbb{T}^{2}\right)$. Motivated by the representation theory of $\mathbb{U}_{t}$ we extend formally the definition of the $V^{n}(K)$ to all integers $n$ by the rules $V^{r}(K)=0, V^{n+2 r}(K)=V^{n}(K)$ and $V^{r+n}(K)=-V^{r-n}(K)$. Here the negative sign means that we color the knot by $V^{r-n}$, then consider the vector with opposite sign in $V\left(\mathbb{T}^{2}\right)$. The following result was proved in [9] using the pairing $[\cdot, \cdot]$ and topological quantum field theory with corners.

Theorem 3.1. In any level $r$ and for any integers $p, q$ and $k$ the following formula holds

$$
C(p, q) V^{k}(\alpha)=t^{-p q}\left(t^{2 q k} V^{k-p}(\alpha)+t^{-2 q k} V^{k+p}(\alpha)\right) .
$$

We will show that the operators of the Weyl quantization act in an identical way on a basis consisting of theta functions. 


\section{The Weyl quantization}

The first general quantization scheme was introduced by Weyl in 1931 . This scheme applies to functions on $\mathbb{R}^{2 n}$ and postulates that the function $e^{2 \pi i\left(p x_{j}+q y_{j}\right)}$ corresponds to the operator $e^{2 \pi\left(p X_{j}+q D_{j}\right)}$ where $X_{j}$ is multiplication by the variable $x_{j}$ and $D_{j}=\frac{1}{2 \pi i} \frac{\partial}{\partial x_{j}}$. In general, the operator associated to a function is the pseudo-differential operator with symbol equal to the function. Since we are quantizing a Kähler manifold, we will convert to the complex picture using the Bargman transform.

Here and throughout the paper we choose for Planck's constant $\hbar=\frac{1}{N}$, where $N=2 r$ is an even integer. This is done so that Weil's integrality condition is satisfied, and so that the Reshetikhin-Turaev topological quantum field theory is well defined. The Hilbert space of the quantization is the space of square integrable holomorphic sections of a line bundle with curvature $N \omega$. It is finite dimensional, as implied by the Heisenberg uncertainty principle since the phase space is compact. It suffices to find the line bundle $\mathcal{L}$ for $N=1$, and then let the bundle for an arbitrary $N$ be $\mathcal{L}^{\otimes N}$.

4.1. The line bundle. The line bundle $\mathcal{L}$ is defined by a cocycle

$$
\chi: \mathbb{C} \times \Lambda \rightarrow \mathbb{C} \backslash\{0\}
$$

as the quotient $\mathbb{C} \times \mathbb{C} / \sim$ under the equivalence $(z, a) \sim(w, b)$ if there is $\lambda \in \Lambda$ such that $(w, b)=(\lambda z, \chi(z, \lambda) a)$. We use the multiplicative notation since the group $\Lambda$ is not commutative. The cocycle condition is

$$
\chi(z, \lambda) \chi(\lambda z, \mu)=\chi(z, \mu \lambda) .
$$

The cocycle is holomorphic off the singular points of the character variety, since the line bundle is. The compatibility with the hermitian structure yields $h(z)=|\chi(z, \lambda)| h\left(\lambda^{-1} z\right)$. To find $\chi$ we first determine $\chi(z, m+i n)$.

Since $h(z+m+i n)=\exp \left(-\frac{\pi}{2}\left(z(m-i n)+\bar{z}(m+i n)+m^{2}+n^{2}\right)\right)$, it follows that

$$
|\chi(z, m+i n)|=\exp \left(\frac{\pi}{2}\left(z(m-i n)+\bar{z}(m+i n)+m^{2}+n^{2}\right)\right) .
$$

From the fact that $\chi$ is holomorphic, it follows that

$$
\chi(z, m+i n)=\exp \left(\pi\left(z(m-i n)+\frac{1}{2}\left(m^{2}+n^{2}\right)\right)\right) \cdot \exp (i \alpha(m, n)) .
$$

The cocycle condition yields

$$
\exp (i \alpha(m, n)+i \alpha(p, q)-i \pi(m q-n p))=\exp (i \alpha(m+p, n+q)) .
$$

This shows that $\exp (\alpha(m, n)-i \pi m n)$ is a morphism from $\mathbb{Z} \times \mathbb{Z}$ to $S^{1}$. We obtain

$$
\begin{array}{r}
\chi(z, m+i n)=(-1)^{m n} \exp \left(\pi\left[z(m-i n)+\frac{1}{2}\left(m^{2}+n^{2}\right)\right]\right) \times \\
\exp (-2 \pi i(\mu m+\nu n))
\end{array}
$$

for some real numbers $\mu$ and $\nu$. 
Recall that $\sigma$ denotes the symmetry of the complex plane with respect to the origin. We have $|\chi(z, \sigma)|=h(z) / h(-z)=1$. Since $\chi$ is holomorphic in $z$ it follows that $\chi(z, \sigma)=\exp (i \pi \beta)$ for some $\beta$. We want to determine $\beta$.

Use the model of the torus obtained by identifying opposite sides of a square. The action of $\sigma$ maps $\frac{1}{2}$ to $-\frac{1}{2}$, and the two correspond to the same point on the character variety. Therefore

$$
\chi\left(\frac{1}{2}, \sigma\right)=\chi\left(\frac{1}{2},-1\right) .
$$

We have

$$
\begin{aligned}
\chi\left(\frac{1}{2},-1\right) & =(-1)^{0} e^{\pi\left(\frac{1}{2}(-1-0 i)+\frac{1}{2}\left((-1)^{2}+0^{2}\right)\right)} e^{-2 \pi i(-\mu+0 \nu)} \\
& =e^{2 \pi i \mu} .
\end{aligned}
$$

Hence $\chi(z, \sigma)=e^{2 \pi i \mu}$, so $\mu=\beta / 2$. The same argument with $\chi\left(\frac{1}{2} i, \sigma\right)$ and $\chi\left(\frac{1}{2} i,-i\right)$ shows that $\nu=\beta / 2$. Also

$$
\chi\left(\frac{1}{2}+\frac{1}{2} i, \sigma\right)=\chi\left(\frac{1}{2}+\frac{1}{2} i,-1-i\right)=e^{\pi i[2(\mu+\nu)-1]}
$$

which implies that modulo $2,2(\mu+\nu)-1=\beta$. Therefore $\beta=1$.

We conclude that

$$
\begin{aligned}
& \chi(z,(m+i n))=(-1)^{m n} \exp \left(\pi\left[z(m-i n)+\frac{1}{2}\left(m^{2}+n^{2}\right)\right]\right) \\
& \chi(z, \sigma)=-1 .
\end{aligned}
$$

4.2. The Hilbert space of the quantization. Recall that the line bundle $\mathcal{L}$ corresponds to the case where the Planck's constant is equal to 1 . To get the general case with $\hbar=1 / N$, we consider the line bundle $\mathcal{L}^{\otimes N}$. This bundle has the hermitian metric defined by $h_{N}(z)=(h(z))^{N}=\exp \left(-\frac{N \pi}{2}|z|^{2}\right)$, and is given by the cocycle $\chi_{N}=(\chi)^{N}$. The Hilbert space of the quantization consists of the sections of the line bundle over $\mathbb{C}$ that are holomorphic and whose pull-backs to the plane are square integrable with respect to the Bargman measure and satisfy $f(\lambda z)=\chi_{N}(z, \lambda) f(z), \lambda \in \Lambda$. The Hilbert space can thus be identified with that of holomorphic square integrable functions on the plane subject to the conditions

$$
f(z+m+i n)=(-1)^{m n N} \exp \left(\pi N\left[z(m-i n)+\frac{1}{2}\left(m^{2}+n^{2}\right)\right]\right) f(z)
$$

and

$$
f(-z)=-f(z)
$$

Here the second condition comes from the action of $\sigma$, hence if we drop it, we obtain the Hilbert space of the quantization of the torus. It can be seen in [5] that the torus has other possible quantization spaces, which arise by twisting the line bundle with flat bundles. This is not the case with $X(\mathbb{Z} \oplus \mathbb{Z})$. 
As suggested by [5] we replace the Hilbert space of the quantization with a space of theta functions. Let

$$
\Theta_{N}=\left\{f \quad \mid \quad f(z+m+i n)=e^{N \pi\left(n^{2}-2 i n z\right)} f(z)\right\}
$$

and let

$$
\mathcal{H}_{N}=\left\{f \in \Theta_{N} \quad \mid \quad f(z)=-f(-z)\right\}
$$

both endowed with the dot product

$$
<f, g>=\int_{\mathbb{T}^{2}} f(z) \overline{g(z)} e^{-2 N \pi y^{2}} d x d y .
$$

Then the Hilbert space of the quantization of the torus is isomorphic to $\Theta_{N}$ via the unitary isomorphism $f(z) \rightarrow e^{-N \pi z^{2} / 2} f(z)$, and the Hilbert space of the quantization of $X(\mathbb{Z} \oplus \mathbb{Z})$ is $\mathcal{H}_{N}$. To be more accurate, the integral that defines the inner product on $\mathcal{H}_{N}$ should be performed over a fundamental domain of the group $\Lambda$, but this gives the same answer as the one above.

Recall that an orthogonal basis of $\Theta_{N}$ is given by $\theta_{j}, j=0,1, \ldots, 2 r-1$, where

$$
\theta_{j}(z)=\sum_{n=-\infty}^{\infty} e^{-\pi\left(N n^{2}+2 j n\right)+2 \pi i z(j+N n)} .
$$

The formula makes sense for all $j$. We have $\theta_{j+N}(z)=e^{\pi(N+2 j)} \theta_{j}(z)$ and $\theta_{-j}(-z)=\theta_{j}(z)$, where the second equality follows by replacing $n$ by $-n$.

An orthonormal basis of $\mathcal{H}_{N}$ is given by

$$
\zeta_{j}=\sqrt[4]{\frac{N}{2}} e^{-\pi j^{2} / N}\left(\theta_{j}-\theta_{-j}\right), \quad j=1,2, \ldots, r-1 .
$$

To see why these vectors are indeed orthonormal note that

$$
\begin{aligned}
& <\theta_{k}-\theta_{-k}, \theta_{j}-\theta_{-j}>=<\theta_{k}, \theta_{j}>+<\theta_{-k}, \theta_{-j}> \\
& -<\theta_{-k}, \theta_{j}>-<\theta_{k}, \theta_{-j}>=\delta_{j k}\left\|\theta_{j}\right\|^{2} .
\end{aligned}
$$

Here we used the fact that the $\theta_{j}$ 's can be shifted to have indices equal to one of the numbers $0,1, \ldots, 2 r-1$ and the latter form an orthonormal basis in the Hilbert space associated to the torus.

Using the same formula we extend the definition of $\zeta_{k}$ for all $k \in \mathbb{Z}$. Clearly $\zeta_{r}$ has to be equal to zero, since

$$
\theta_{r}(z)-\theta_{-r}(z)=\theta_{r}(z)-e^{-\pi(2 r-2 r)} \theta_{r}(z)=0 .
$$

Also, for $1 \leq k \leq r-1$ we have

$$
\begin{aligned}
\theta_{r+k}-\theta_{-r-k} & =e^{\pi(2 r-2(-r+k))} \theta_{-r+k}-e^{-\pi(2 r+2(r-k))} \theta_{r-k} \\
& =-e^{2 r k \pi}\left(\theta_{r-k}-\theta_{-r+k}\right) .
\end{aligned}
$$

Thus normalizing we get that $\zeta_{r+k}=-\zeta_{r-k}$. Finally, since $\theta_{j+2 r}$ is a multiple of $\theta_{j}$ it follows that $\zeta_{j+2 r}=\zeta_{j}$, for all integers $j$. 
4.3. The operators of the quantization. Each observable $f: X(\mathbb{Z} \oplus$ $\mathbb{Z}) \rightarrow \mathbb{R}$ yields a sequence of operators indexed by the level $N=2 r$. Whenever there is no danger of confusion we omit the index $N$. We relate Weyl quantization to Toeplitz quantization and then work with Toeplitz operators, for which computations are easier.

Let us first consider the case of the complex plane. Modulo some adjustments to suit the notations of this paper, Propositions 2.96 and 2.97 in [7] (see also [12]) show that the operator associated by the Toeplitz quantization to a function $f$ on $\mathbb{C}$ is equal to the operator associated by the Weyl quantization to the function

$$
\sigma(z, \bar{z})=\frac{1}{N} \int_{\mathbb{C}} e^{-2 \pi|z-u|^{2} N} f(u, \bar{u}) d u d \bar{u} .
$$

Note that

$$
\sigma(z, \bar{z})=e^{\frac{\Delta}{4 N}} f(z, \bar{z})
$$

where

$$
\Delta f=\frac{1}{2 \pi}\left(\frac{\partial^{2} f}{\partial x^{2}}+\frac{\partial^{2} f}{\partial y^{2}}\right)
$$

Here we used the notation $z=x+i y, \bar{z}=x-i y$. Hence doing Weyl quantization with symbol $f$ is the same as doing Toeplitz quantization with symbol $e^{-\frac{\Delta}{4 N}} f$. Thinking equivariantly, we now define the operators of the quantization of the character variety. Let

$$
\Pi_{N}: L^{2}\left(X(\mathbb{Z} \oplus \mathbb{Z}), \mathcal{L}^{\otimes N}\right) \rightarrow \mathcal{H}_{N}
$$

be the orthogonal projection from the space of square integrable sections with respect to the measure $e^{-2 N \pi y^{2}} d x d y$ onto the space $\mathcal{H}_{N}$.

To a $C^{\infty}$ function $f$ on the character variety we associate the operator $o p_{N}(f)$ (in level $N$ ) given by

$$
o p_{N}(f): \mathcal{H}_{N} \rightarrow \mathcal{H}_{N}, \quad g \rightarrow \Pi_{N}\left(\left(e^{-\frac{\Delta}{4 N}} f\right) g\right) .
$$

The operator $g \rightarrow \Pi_{N}(f g)$ is the Toeplitz operator of symbol $f$, denoted by $T_{f}$.

An important family of operators are the ones associated to the functions $2 \cos 2 \pi(p x+q y)$, which we denote by $\underline{C}(p, q)$. These are the same as the Toeplitz operators with symbols

$$
2 e^{-\frac{\Delta}{4 N}} \cos 2 \pi(p x+q y)=2 e^{\frac{p^{2}+q^{2}}{2 N} \pi} \cos 2 \pi(p x+q y) .
$$

\section{WEYL QUANTIZATION VERSUS QUANTUM GROUP QUANTIZATION}

To simplify the computation, we pull back everything to the line bundle on the torus. Hence we do the computations in $\Theta_{N}$. We start with two lemmas that hold on the torus. They were inspired by [5]. 
Let $j, k, p$ be integers such that $-r+1 \leq j, k \leq r-1, p=p_{0}+\gamma N$ with $\gamma$ an integer. There are two possibilities $-r+1 \leq j+p_{0}<N=2 r$ or $N=2 r \leq j+p_{0}<N+1$. Let also $u(y)$ be a bounded continuous function.

Lemma 5.1. Assume that $j+p_{0}<N$. Then $<e^{2 \pi i p x} u(y) \theta_{j}, \theta_{k}>$ is different from zero if and only if $k=j+p_{0}$ and in this case it is equal to

$$
e^{-\frac{\pi}{2 N} p^{2}+\left(j+p_{0} / 2\right)^{2} / N} \sum_{m=-\infty}^{\infty} e^{-\pi m^{2} / 2 N} e^{-2 \pi i(j+p / 2) m / N} \hat{u}(m)
$$

where $\hat{u}(m)$ is the mth Fourier coefficient of $u$.

Proof. Separating the variables we obtain

$$
\begin{aligned}
< & \exp (2 \pi p x) u(y) \theta_{j}, \theta_{k}>=\int_{\mathbb{T}^{2}} \exp (2 \pi p x) u(y) \theta_{j} \overline{\theta_{k}} e^{-2 N \pi y^{2}} d x d y \\
= & \sum_{m, n} e^{-\pi\left(N m^{2}+2 j m+N n^{2}+2 k n\right)} \int_{0}^{1} e^{2 \pi i x(N(m-n)+p+j-k)} d x \\
& \times \int_{0}^{1} e^{-2 \pi y(j+N n+k+N m)-2 \pi N y^{2}} u(y) d y .
\end{aligned}
$$

The first integral is equal to zero unless $n=m+\gamma$ and $k=p_{0}+j$. If $k=p_{0}+j$ the expression becomes

$$
\begin{aligned}
& e^{-\pi N \gamma^{2}-2 \pi j \gamma-2 \pi p_{0} \gamma} \int_{0}^{1}\left(e^{-2 \pi\left(N y^{2}+\left(2 j+p_{0}+N \gamma\right) y\right)}\right. \\
& \left.\quad \times \sum_{m} e^{-2 \pi\left(N m^{2}+N m \gamma+2 m\left(j+\frac{p_{0}}{2}+N y\right)\right)}\right) u(y) d y .
\end{aligned}
$$

After completing the square in the exponent of the third exponential we obtain that this is equal to

$$
e^{-\frac{\pi}{2 N} p^{2}+\left(j+p_{0} / 2\right)^{2} / N} \int_{0}^{1} \sum_{m} e^{-2 \pi N\left(m+y+\frac{j+p / 2}{N}\right)^{2}} u(y) d y .
$$

Using the Poisson formula $\left(\sum_{m} f(m)=\sum_{m} \hat{f}(m)\right)$ for the function $e^{-x^{2}}$ we transform the sum of the exponentials into

$$
\sum_{m} e^{-\pi m^{2} / 2 N} e^{2 \pi i\left(y+\frac{j+p / 2}{N}\right) m} .
$$

It follows that the inner product we are computing is equal to

$$
e^{-\frac{\pi}{2 N} p^{2}+\pi\left(j+p_{0} / 2\right)^{2} / N} \sum_{m} e^{-\pi m^{2} / 2 N} e^{2 \pi i(j+p / 2) m / N} \int_{0}^{1} e^{2 \pi i m y} u(y) d y
$$

which proves the lemma. 
Lemma 5.2. Assume that $j+p_{0} \geq N$ and denote $p_{1}=p-(\gamma+1) N$. Then $<e^{2 \pi i p x} u(y) \theta_{j}, \theta_{k}>$ is different from zero if and only if $k=j+p_{1}$ and in this case it is equal to

$$
e^{-\frac{\pi}{2 N} p^{2}+\pi\left(j+p_{1} / 2\right)^{2} / N} \sum_{n=-\infty}^{\infty} e^{-\pi m^{2} / 2 N} e^{-2 \pi i(j+p / 2) m / N} \hat{u}(m)
$$

where $\hat{u}(m)$ is the mth Fourier coefficient of $u$.

Proof. We start with a computation like the one in the proof of Lemma 1 to conclude that $k=p_{0}+j-N=p_{1}+j$ and $m=n-\gamma-1$. From here the same considerations apply mutatis mutandis to yield the conclusion.

Theorem 5.3. There exists a unitary isomorphism between $V\left(\mathbb{T}^{2}\right)$ and $\mathcal{H}_{N}$ sending $V^{j}(\alpha)$ to $\zeta_{j}, j=1,2, \ldots, r-1$, which transforms the operator associated through quantum group quantization to the function $f$ into the operator associated by Weyl quantization to the same function.

Proof. We verify that the matrix of the operator $\underline{C}(p, q)$ in the basis $\zeta_{j}$ is the same as the matrix of the operator $C(p, q)$ in the basis $V^{j}(\alpha)$. We have

$$
\underline{C}(p, q)=e^{\frac{p^{2}+q^{2}}{2 N}} T_{2 \cos 2 \pi(p x+q y)}
$$

where $T_{2 \cos 2 \pi(p x+q y)}$ is the Toeplitz operator of symbol $2 \cos 2 \pi(p x+q y)$. Let us pull back everything to the torus using the covering map $\mathbb{T}^{2} \rightarrow X(\mathbb{Z} \oplus \mathbb{Z})$ so that we can work with exponentials.

We do first the case $j+p_{0}<N$. If in Lemma 5.1 we let $u(y)=e^{2 \pi i q y}$ we obtain

$$
T_{e^{2 \pi i p x+2 \pi i q y}} \theta_{j}=e^{-\frac{\pi}{2 N} p^{2}+\left(j+p_{1} / 2\right)^{2} / N} e^{-\pi q^{2} / 2 N} e^{-2 \pi i(j+p / 2) q / N} \theta_{j+p_{0}} .
$$

Using this formula and the fact that

$$
\zeta_{j}=\sqrt[4]{\frac{N}{2}} e^{-\pi j^{2} / N}\left(\theta_{j}-\theta_{-j}\right)
$$

after doing the algebraic computations we arrive at

$$
e^{\frac{p^{2}+q^{2}}{2 N} \pi} T_{2 \cos 2 \pi(p x+q y)} \zeta_{j}=t^{-p q}\left(t^{2 j q} \zeta_{j-p_{0}}+t^{-2 j q} \zeta_{j+p_{0}}\right) .
$$

If $j+p_{0} \geq N$, we let $p_{1}=p-(\gamma+1) N$, and an application of Lemma 5.2 shows that in this case

$$
e^{\frac{p^{2}+q^{2}}{2 N} \pi} T_{2 \cos 2 \pi(p x+q y)} \zeta_{j}=t^{-p q}\left(t^{2 j q} \zeta_{j-p_{1}}+t^{-2 j q} \zeta_{j+p_{1}}\right) .
$$

But we have seen that $\zeta_{j+N}=\zeta_{j}$ for all $j$, so in the above formulas $p_{0}$ and $p_{1}$ can be replaced by $p$. It follows that for all $j, 1 \leq j \leq r-1$, and all integers $p$ and $q$ we have

$$
e^{\frac{p^{2}+q^{2}}{2 N} \pi} T_{2 \cos 2 \pi(p x+q y)} \zeta_{j}=t^{-p q}\left(t^{2 j q} \zeta_{j-p}+t^{-2 j q} \zeta_{j+p}\right) .
$$

Theorem 3.1 shows that

$$
C(p, q) V^{j}(\alpha)=t^{-p q}\left(t^{2 j q} V^{j-p}(\alpha)+t^{-2 j q} V^{j+p}(\alpha)\right) .
$$


Hence the unitary isomorphism defined by $V^{j}(\alpha) \rightarrow \zeta_{j}$ transforms the operator $C(p, q)$ into the operator $\underline{C}(p, q)$, and the theorem is proved.

From now on we identify the two quantizations and use the notation $C(p, q)$ for the operators. Recall the notation $t=e^{i \pi / N}$. As a byproduct of the proof of the theorem we obtain the following product-to-sum formula for $C(p, q)$ 's, which was already noticed in [9].

Proposition 5.4. For any integers $m, n, p, q$ one has

$$
C(m, n) * C(p, q)=t^{\mid m q}{ }^{m n} \mid C(m+p, n+q)+t^{-\left|{ }_{p q}^{m n}\right|} C(m-p, n-q),
$$

where ||$_{p q}^{m n} \mid$ is the determinant.

We conclude this section by noting that in Witten's picture the operator associated by Weyl quantization to the function $\sin 2 \pi(n+1)\left(p^{\prime} x+\right.$ $\left.q^{\prime} y\right) / \sin 2 \pi\left(p^{\prime} x+q^{\prime} y\right)\left(p^{\prime}, q^{\prime}\right.$ relatively prime) is the same as the quantum group quantization of the Wilson line around the curve of slope $p^{\prime} / q^{\prime}$ on the torus in the $n$-dimensional irreducible representation of $S U(2)$.

\section{THE STAR PRODUCT}

6.1. Definition of the star product. Let $(M, \omega)$ be a symplectic manifold. A $*$-product on $M$ is a binary operation on

$$
C^{\infty}(M)\left[\left[N^{-1}\right]\right]
$$

which is associative, and for all $f, g \in C^{\infty}(M)$ satisfies $N^{-k} f * g=f *$ $N^{-k} g=N^{-k}(f * g)$ and also

$$
f * g=\sum_{k=0}^{\infty} N^{-k} B_{k}(f, g) .
$$

The operators $B_{k}(f, g)$ are bi-differential operators from $C^{\infty}(M) \times C^{\infty}(M)$ to $C^{\infty}(M)$, such that $B_{0}(f, g)=f g$, and such that Dirac's correspondence principle

$$
B_{1}(f, g)-B_{1}(g, f)=\{f, g\}
$$

is satisfied. Here $\{f, g\}$ stands for the Poisson bracket induced by the symplectic form. One says that $C^{\infty}(M)\left[\left[N^{-1}\right]\right]$ is a deformation of $C^{\infty}(M)$ in the direction of the given Poisson bracket. We use $N$ for the variable of the formal series to be consistent with the rest of the paper.

The character variety $X(\mathbb{Z} \oplus \mathbb{Z})$ is a symplectic manifold off the four singularities.

Proposition 6.1. The formula

$$
\begin{aligned}
& 2 \cos 2 \pi(m x+n y) * 2 \cos 2 \pi(p x+q y) \\
& =t_{p q}^{\mid m n} \mid 2 \cos 2 \pi((m+p) x+(n+q) y) \\
& \quad+t^{-||_{p q}^{m n} \mid} 2 \cos 2 \pi((m-p) x+(n-q) y)
\end{aligned}
$$


defines a *-product on $C^{\infty} X(\mathbb{Z} \oplus \mathbb{Z})(M)\left[\left[N^{-1}\right]\right]$, which is a deformation quantization in the direction of the Kähler form $i \pi d z \wedge d \bar{z}$.

In these formulas the exponentials should be expanded formally into power series in $N^{-1}$.

Proof. We have

$$
\begin{aligned}
& 2 \cos 2 \pi(m x+n y) * 2 \cos 2 \pi(p x+q y) \\
& \quad-2 \cos 2 \pi(m x+n y) * 2 \cos 2 \pi(p x+q y)= \\
& \pi(i m q-i n p) N^{-1} 2 \cos 2 \pi((m+p) x+(n+q) y) \\
& +\pi(i n p-i m q) N^{-1} 2 \cos 2 \pi((m-p) x+(n-q) y) \\
& -\pi(i p n-i q m) N^{-1} 2 \cos 2 \pi((p+m) x+(q+n) y) \\
& -\pi(i m q-i n p) N^{-1} 2 \cos 2 \pi((p-m) x+(q-n) y)+O\left(N^{-2}\right) \\
& =2 \pi i N^{-1}(m q-n p) 2 \cos 2 \pi((m+p) x+(n+q) y) \\
& -2 \pi i N^{-1}(m q-n p) 2 \cos 2 \pi((m-p) x+(n-q) y)+O\left(N^{-2}\right) \\
& =N^{-1}\{2 \cos 2 \pi(m x+n y), 2 \cos 2 \pi(p x+q y)\}+O\left(N^{-2}\right) .
\end{aligned}
$$

so the correspondence principle is satisfied. The coefficients $B_{k}(f, g)$ are bidifferential operators since

$$
\left.B_{1}(f, g)=\frac{1}{4 \pi i} \operatorname{det}\left|\begin{array}{cc}
\frac{\partial}{\partial x_{1}} & \frac{\partial}{\partial y_{1}} \\
\frac{\partial}{\partial x_{2}} & \frac{\partial}{\partial y_{2}}
\end{array}\right| f\left(x_{1}, y_{1}\right) g\left(x_{2}, y_{2}\right)\right) \begin{aligned}
& x_{1}=x_{2}=x \\
& y_{1}=y_{2}=y
\end{aligned}
$$

and $B_{k}=B_{1}^{k} / k !$.

We would like to point out that this $*$-product is different from the one that would arise if we applied the quantization methods outlined in [3] since Berezin's ideas correspond to the anti-normal (respectively normal) ordering of the operators.

Thinking now of the deformation parameter as a fixed natural number we see that the $*$-algebra defined in Proposition 6.1 is a subalgebra of Rieffel's noncommutative torus [17] with rational Planck's constant. That is, our *-product is the restriction of Rieffel's *-product to trigonometric series in cosines.

6.2. The generalized Hardy space. In 11] Guillemin has shown that for each compact symplectic prequantizable manifold $M$ there exists a *product and a circle bundle $S^{1} \hookrightarrow P \rightarrow M$ with a canonical volume $\mu$ such that $L^{2}(P, \mu)$ contains finite dimensional vector spaces $\mathcal{H}_{N}$ of $N$-equivariant functions $\left(\bmod\right.$ the action of $\left.S^{1}\right)$ satisfying for all $f, g \in C^{\infty}(M)$ :

$$
\Pi_{N} M_{f} \Pi_{N} M_{g} \Pi_{N}=\Pi_{N} M_{(f * g)_{N}} \Pi_{N}+O\left(N^{-\infty}\right) .
$$

where $\Pi_{N}$ is the orthogonal projection onto $\mathcal{H}_{N}, M_{f}$ is the multiplication by $f$ and $(f * g)_{N}$ is to be understood as the $*$-product for a certain fixed integer value of $N$. 
Our operators are not Toeplitz so they won't fit exactly Guillemin's construction. However, along the same lines we will construct a representation of the $*$-algebra from the previous section onto an infinite dimensional Hilbert space that contains all $\mathcal{H}_{N}$ 's as direct summands. The reader can find a detailed account on how these things are done in general in [6].

Let $\mathcal{L}$ be the line bundle constructed in 4.1 , and let $Z \subset \mathcal{L}^{*}$ be the unit circle bundle in the dual of $\mathcal{L}$. $Z$ is an $S^{1}$-principal bundle. A point in $Z$ is a pair $(x, \phi)$, where $x \in \mathbb{C}$ and $\phi$ is a complex valued functional with $|\phi(x)|=\|x\|$ (the length of $x$ being given by the hermitian structure). More precisely

$$
Z=\left\{(z, \xi) ; \quad|\xi|=e^{-\pi|z|^{2} / 2}\right\} .
$$

The map $(z, \xi) \rightarrow(z, \xi /|\xi|)$ identifies $Z$ with $\mathbb{C} \times S^{1}$. Let $\theta$ be the argument of $\xi$ and consider the volume form $d \theta d z$ on $Z$. Using this volume form we can define the space $L^{2}(Z)$.

Now let $N$ be an even integer. Note that

$$
\mathcal{L}^{\otimes N} \simeq Z \times_{N} \mathbb{C}
$$

where $Z \times{ }_{N} \mathbb{C}$ is the quotient of $Z \times \mathbb{C}$ by the equivalence

$$
\left(p \cdot e^{i N \theta}, z\right) \sim\left(p, e^{i N \theta} z\right) .
$$

A complex valued smooth function $f$ on $Z$ is called $N$-equivariant if for all $(x, \phi) \in Z$,

$$
f\left(x, \phi \cdot e^{i \theta}\right)=e^{i N \theta} f(x, \phi) .
$$

The set of all $N$-equivariant functions is denoted by $C^{\infty}(Z)_{N}$. There exists an isomorphism

$$
C^{\infty}\left(X(\mathbb{Z} \oplus \mathbb{Z}), \mathcal{L}^{\otimes N}\right) \simeq C^{\infty}(Z)_{N},
$$

which transforms a section $s \in C^{\infty}\left(X(\mathbb{Z} \oplus \mathbb{Z}), \mathcal{L}^{\otimes N}\right)$ to a function $f$, with $f(z, \xi)=\xi^{N} s(z)$. Here of course $s$ has to be viewed as a $\mathbb{C}$-valued function subject to the equivariance conditions from Section 4 . It is easy to see that this map gives rise to a unitary isomorphism between the space of $L^{2}$ sections of $\mathcal{L}^{\otimes N}$ and the $L^{2}$-completion of $C^{\infty}(Z)_{N}$. A little Fourier analysis (involving integrals of the form $\int e^{i m \theta} e^{-i n \theta} d \theta$ ) shows that for different $N$ 's, the images of the corresponding $L^{2}$ spaces are orthogonal.

As a result, the spaces $\mathcal{H}_{N}$ are embedded as mutually orthogonal subspaces of $L^{2}(Z)$. Define

$$
\mathcal{H}=\bigoplus_{N \text { even }} \mathcal{H}_{N}
$$

This space is the generalized version of the classical Hardy space.

We denote by $\Pi$ the orthogonal projection of $L^{2}(Z)$ onto $\mathcal{H}$. Let $f$ be a smooth function on $X(\mathbb{Z} \oplus \mathbb{Z})$, which can be viewed as as the limit (in the 
$C^{\infty}$ topology) of a sequence of trigonometric polynomials in $\cos (p x+q y)$, $p, q \in \mathbb{Z}$. Define the operator

$$
o p(f): \mathcal{H} \rightarrow \mathcal{H}, \quad g \rightarrow \Pi\left(\left(e^{-\frac{\Delta}{4 N}} f\right) g\right) .
$$

If $e^{-\frac{\Delta}{4 N}} f$ is a $C^{\infty}$ function on the character variety, this this operator is bounded. The restriction of $o p(f)$ to $\mathcal{H}_{N}$ coincides with $o p_{N}(f)$, for all $N \geq 1$. Moreover, the product-to-sum formula from Proposition 5.4 shows that the $*$-product on $C^{\infty}(X(\mathbb{Z} \oplus \mathbb{Z}))\left[\left[N^{-1}\right]\right]$ defined by the multiplication of these operators is the same as the $*$-product introduced in Section 6.1.

\section{FinAL REMARKS}

An alternative approach to the Reshetikhin-Turaev theory was constructed in [4] using Kauffman bracket skein modules. This approach also leads to a quantization of the moduli space of flat $S U(2)$ connections on the torus. Do we obtain the Weyl quantization in that situation as well? The answer is no.

Indeed, the analogues of the basis vectors $V^{n}(\alpha)$ are the colorings of the core of the solid torus by Jones-Wenzl idempotents. More precisely, to the vector $V^{n}(\alpha)$ corresponds the vector $S_{n-1}(\alpha)$, where $S_{n-1}(\alpha)$ is the coloring of $\alpha$ by the $n-1$ st Jones-Wenzl idempotent. It follows from Theorem 5.6 and the discussion preceding it in [8] that the action of the operator associated to $2 \cos 2 \pi(p x+q y)$, denoted by $(p, q)_{T}$, on these basis elements is

$$
(p, q)_{T} S_{n-1}(\alpha)=(-1)^{q} t^{-p q}\left(t^{2 q k} S_{k-p-1}(\alpha)+t^{-2 q k} S_{k+p-1}(\alpha)\right) .
$$

The factor $(-1)^{q}$ does not appear in the formula from Theorem 3.1, proving that this quantization is different. However, both quantizations yield the same $*$-algebra, as shown in [8].

Finally, although quantum field theory is intimately related to Wick quantization, the present paper shows that this is not the case with the topological quantum field theory of Reshetikhin and Turaev.

\section{REFERENCES}

[1] M.F. Atiyah, The Geometry and Physics of Knots, Lezioni Lincee, Cambridge Univ. Press, 1990.

[2] M.F. Atiyah, R. Bott, The Yang-Mills equations over a Riemann surface, Phil. Tras. Royal Soc. A 308(1982), 523-.

[3] F.A. Berezin, Quantization, Math. USSR Izvestija, 8(1974), 1109-1165.

[4] C. Blanchet, N. Habegger, G. Masbaum, P. Vogel, Topological quantum field theories derived from the Kauffman bracket, Topology 34(1995), 883-927.

[5] A. Bloch, F. Golse, T. Paul, A. Uribe, Dispersionless Toda and Toeplitz operators, preprint.

[6] D. Borthwick, Introduction to Kähler quantization, in First Summer School in Analysis and Mathematical Physics, Cuernavaca Morelos, Mexico, Contemporary Math. $\mathbf{2 6 0}(2000)$, 91-132.

[7] G. Folland, Harmonic Analysis in Phase Space, Princeton University Press, Princeton 1989. 
[8] Ch. Frohman, R. Gelca, Skein modules and the noncommutative torus, Transactions Amer. Math. Soc., 352(2000), 4877-4888.

[9] R. Gelca, A product-to-sum formula for the quantum group of $S L(2, \mathbb{C})$, preprint.

[10] W. Goldman, Invariant functions on Lie groups and Hamiltonian flow of surface group representations, Inventiones Math., 85(1986), 263-302.

[11] V. Guillemin, Star Products on Compact Pre-quantizable Symplectic Manifolds, Lett. Math. Phys., 35(1995), 85-89.

[12] B.C. Hall, Holomorphic methods in analysis and mathematical physics in First Summer School in Analysis and Mathematical Physics, Cuernavaca Morelos, Mexico, Contemporary Math. 260(2000), 1-59.

[13] V.F.R. Jones, Polynomial invariants of knots via von Neumann algebras, Bull. Amer. Math. Soc., 12(1985), 103-111.

[14] A.N. Kirillov, N.Y. Reshetikhin, Representation of the algebra $U_{q}\left(s l_{2}\right)$, q-orthogonal polynomials and invariants of links, in: Infinite dimensional Lie algebras and groups, (ed. by V.G. Kac), Adv. Ser. in Math. Phys. 7, World Scientific, Singapore 1988, 285-339.

[15] N.Yu. Reshetikhin, L.A. Takhtajan, Deformation Quantization of Kähler manifolds, preprint.

[16] N.Yu. Reshetikhin, V.G. Turaev, Invariants of 3-manifolds via link polynomials and quantum groups, Inventiones Math., 103(1991), 547-597.

[17] M. Rieffel, Deformation quantization of Heisenberg Manifolds, Commun. Math. Phys. 122(1989, 531-562.

[18] V.G. Turaev, Quantum Invariants of Knots and 3-Manifolds, de Gruyter, 1994.

[19] J. Weitsman, Quantization via real polarization of the moduli space of flat connections and Chern-Simons gauge theory in genus one, Commun. Math. Phys., 137(1991), 175-190

[20] E. Witten, Quantum field theory and the Jones polynomial, Comm. Math. Phys., 121(1989), 351-399.

Department of Mathematics and Statistics, Texas Tech University, LubBock, TX 79409 and Institute of Mathematics of the Romanian Academy, BuChaREST, ROMANIA

E-mail address: rgelca@math.ttu.edu

Department of Mathematics, University of Michigan, Ann Arbor, Mi 48109

E-mail address: uribe@math.1sa.umich.edu 